\title{
miR-590 promotes the proliferation of HUMSCs and induces ECM synthesis by targeting Smad7
}

\author{
TIANMING LIU*, YINGE WU*, TENGYI HUANG, XUEXUAN ZHANG and YINGMU CAI \\ Clinical Medical Laboratory, The First Affiliated Hospital of Shantou University Medical College, \\ Shantou, Guangdong 515041, P.R. China
}

Received October 19, 2015; Accepted March 23, 2017

DOI 10.3892/ol.2017.6663

\begin{abstract}
MicroRNA (miR)-590 has been established to be a promoter of cell proliferation, migration and invasion, and an inhibitor of apoptosis in numerous cancer cell lines. However, its effects on non-cancer cells remain to be elucidated. miR-590 was transfected into human umbilical cord mesenchymal stem cells (HUMSCs), and the cell proliferation rate was determined using a Cell-Light 5-ethynyl-20-deoxyuridine Apollo 567 kit and the presence of extracellular matrix (ECM) proteins were detected using western blot analysis and immunofluorescence microscopy. Using bioinformatic analysis and dual-luciferase assays, the novel target miR-590 was identified. In addition, the effects of miR-590 on cell proliferation and ECM enhancement were also evaluated. The results of the present study demonstrated that miR-590 interacts directly with the 3'-untranslated region of Mothers Against Decapentaplegic Homolog 7 (Smad7), which is an important factor in transforming growth factor- $\beta$ signaling pathway. Overexpression of miR-590 downregulated Smad7 expression at the mRNA and protein level, and subsequently resulted in cell proliferation and ECM accumulation. Additionally, the transfection of small interfering RNA targeting Smad7 in HUMSCs produced similar effects on cell proliferation and ECM to the overexpression of miR-590. The results of the present study indicated that miR-590 affects HUMSC proliferation by directly targeting Smad7.
\end{abstract}

\section{Introduction}

Tissue trauma is a typical pathological disease in which normal tissue undergoes structural destruction resulting in

Correspondence to: Professor Yingmu Cai, Clinical Medical Laboratory, The First Affiliated Hospital of Shantou University Medical College, 57 Changping Road, Shantou, Guangdong 515041, P.R. China

E-mail: st_ycaiyingmu@sina.com

*Contributed equally

Key words: miR-590, Mothers Against Decapentaplegic Homolog 7, human umbilical cord mesenchymal stem cells, cell proliferation, extracellular matrix functional impairment (1). Therefore, rapid wound healing is an important topic for further investigation. An established hypothesis is to use stem cells to repair the destroyed tissue (2). Mesenchymal stem cells (MSCs) obtained from Wharton's jelly are adult stem cells that have multiple differentiation pathways and self-renewal potential. Compared with other tissue-derived stem cells, the umbilical cord is readily available and has limited immunogenicity and bioethical considerations (3). In addition, previous studies have identified that microRNA (miRNA/miR) may serve an important role in wound healing $(4,5)$. However, the role of miRNAs in human umbilical cord mesenchymal stem cells (HUMSCs) for wound healing remains to be elucidated.

miRNAs are a type of small non-coding RNA of between 19 and 25 nucleotides in length, which regulate gene expression through targeting the 3'-untranslated region (3'-UTR) of mRNA resulting in translation repression or degradation (6). miR-590 has been identified as a tumor promoter by repressing proliferation-associated genes and enhancing cell growth in renal carcinoma cancer (7), hepatocellular carcinoma (8), acute myeloid leukemia (9), cervical cancer (10) and lung cancer (11). According to previous studies, eukaryotic translation elongation factor $1 \varepsilon 1$ (12), transforming growth factor (TGF) $\beta$ receptor II (8), S100 calcium-binding protein A10 (9), polybromo 1 (7) and cell adhesion molecule with homology with L1 cell adhesion molecule (10) are targets of miR-590. The downregulated expression of miR-590 induces fibrosis in nicotine-induced atrial fibrosis (13); however, the potential effects of miR-590 on cell proliferation and extracellular matrix (ECM) synthesis are poorly understood in stem cells. Smad7 has been established to serve important roles in the TGF $\beta$ signaling pathway (14). Previous studies have demonstrated that Smad7 is a vital factor in adult homoeostasis and embryonic development, and abnormal expression of Smad7 is associated with human diseases via ECM synthesis and the development of tumors $(15,16)$. However, the role of miRNA/Smad7 regulation in traumatic disease is poorly understood. Jia et al (17) demonstrated that miR-17-5p regulated osteoblastic differentiation and proliferation by targeting Smad7, and Liu et al (18) identified that miR-21 affected the ECM deposit in lung by targeting Smad7. In addition, miR-195 (19), miR-16 (20), miR-132 (21), miR-15 (22) and miR-92a (23) regulate Smad7 expression levels. The results of the present study demonstrate that miR-590 promotes the 
proliferation of HUMSCs and induces ECM expression in HUMSCs by targeting Smad7. miR-590 may be a key regulator in stem cell growth and tissue repair.

\section{Materials and methods}

Cell culture. A HUMSC human stem cell line, was purchased from the American TypeCulture Collection (cat.no.CSC-7700W; Manassas, VA, USA) and cultured in MSC growth medium (Gibco; Thermo Fisher Scientific, Inc., Waltham, MA, USA) supplemented with low-glucose Dulbecco's modified Eagle's medium (HyClone; GE Healthcare Life Sciences, Logan, UT, USA) plus $10 \%$ heat-inactivated fetal bovine serum (HyClone; GE Healthcare Life Sciences), $100 \mathrm{U} / \mathrm{ml}$ penicillin and $100 \mathrm{U} / \mathrm{ml}$ streptomycin (Beyotime Institute of Biotechnology, Haimen, China). Cells were cultured in a humidified incubator at $37^{\circ} \mathrm{C}$ in at atmosphere containing $5 \% \mathrm{CO}_{2}$.

Transfection. The miR-590 mimic and small interfering RNAs (siRNAs) were synthesized by Guangzhou RiboBio Co., Ltd. (Guangzhou, China). The oligonucleotide sequence of Smad7 siRNA was as follows: 5'-AAGCUCAAUUCGGAC AACAAG-3'. miRNA mimic and siRNA transfections were performed using Lipofectamine ${ }^{\circledR} 2000$ (Invitrogen; Thermo Fisher Scientific,Inc.), according to the manufacturer's protocol. A total of 50 or $100 \mathrm{nM}$ miR-590 mimic or negative control, and $50 \mathrm{nM}$ siRNA, were used for transfection in Opti-MEM serum-free medium (Gibco; Thermo Fisher Scientific, Inc.). A total of $5 \times 10^{6}$ HUMSCs were plated in each well of 24-well plates (Invitrogen; Thermo Fisher Scientific, Inc.). Total RNA and protein were prepared at 36 or $48 \mathrm{~h}$ respectively following transfection for further experiments.

Quantitative analysis of miRNAs and mRNAs. Total RNA was extracted from cultured HUMSCs using TRizol (Invitrogen; Thermo Fisher Scientific, Inc.), according to the manufacturer's protocol. The reverse transcription-quantitative polymerase chain reaction (RT-qPCR) was used to analyze the expression of miR-590 with primers from Guangzhou RiboBio Co., Ltd., and U6 was selected as internal reference. For quantitative analysis of Smad7 expression, 500 ng total RNA was used for the synthesis of random-primed single-stranded cDNA using a First-Strand cDNA Synthesis kit (Thermo Fisher Scientific, Inc.) and qPCR was performed using SYBR-Green S Mixture (CWBIO, Beijing, China). The relative quantity of Smad7 was normalized to $18 \mathrm{~S}$ as per a previously described method (24). The thermocycling conditions were; pre-degeneration at $95^{\circ} \mathrm{C}$ for $5 \mathrm{~min}$, degeneration at $94^{\circ} \mathrm{C}$ for $20 \mathrm{sec}$, annealing at $56^{\circ} \mathrm{C}$ for $30 \mathrm{sec}$, extension at $72^{\circ} \mathrm{C}$ for $10 \mathrm{sec}(25 \mathrm{cycles})$, extension at $72^{\circ} \mathrm{C}$ for $2 \mathrm{~min}$ and a final round at $16^{\circ} \mathrm{C}$ for $5 \mathrm{~min}$. The sequence of PCR primers were as follows: $18 \mathrm{~S}$ forward, 5'-GTAACCCGTTGAACCCCATT-3'; 18S reverse, 5'-CCA TCCAATCGGTAGTAGCG-3'; Smad7 forward, 5'-CCTCCT TACTCCAGATACC-3'; Smad7 reverse, 5'-GTCTTCTCC TCCCAGTATG-3'; U6 forward, 5'-CTCGCTTCGGCAGCA CA-3', U6 reverse, 5'-AACGCTTCACGAATTTGCGT-3'. Three independent experiments were performed.

Protein extraction and western blot analysis. At $48 \mathrm{~h}$ after transfection, HUMSCs were lysed using radioimmunoprecipitation lysis buffer containing $2 \mathrm{mM}$ phenylmethylsulfonyl fluoride (Sigma-Aldrich; Merck KGaA, Darmstadt, Germany). Each sample protein concentration was determined using a Bicinchoninic Protein Assay kit (Beyotime Institute of Biotechnology) and equal amounts of total protein $(80 \mu \mathrm{g})$ were separated by SDS-PAGE (12\% gels) and transferred onto polyvinylidene membranes (Merck KGaA). Membranes were blocked with 5\% non-fat milk in Tris-buffered saline containing $0.05 \%$ Tween- 20 for $1 \mathrm{~h}$ at room temperature, incubated overnight with primary antibody at $4^{\circ} \mathrm{C}$, washed three times with 1X TBST buffer for $5 \mathrm{~min}$ each time at room temperature, incubated with secondary antibody for $1 \mathrm{~h}$ at room temperature and finally visualized by Chemiluminescence Horseradish Peroxidase (HRP) Substrate reagent (Merck KGaA). The antibodies used in the present study were as follows: Mouse anti- $\beta$-tubulin (HC101; 1:5,000; Beijing Transgen Biotech Co., Ltd., Beijing, China), rabbit anti-Smad7 (25840-1-AP; 1:4,000; ProteinTech Group, Inc., Chicago, IL, USA), rabbit anti-collagen I (ab209539; 1:4,000; Abcam, Cambridge, UK), rabbit anti-fibronectin (FN) (ab6584; 1:4,000; Abcam), HRP-labeled goat anti-mouse Immunoglobulin $\mathrm{G}$ ( $\mathrm{IgG} ; 10004302-1$; 1:5,000; Beijing Zhongshan Jinqiao Biotechnology Co., Ltd., Beijing, China) and HRP-labeled goat anti-rabbit IgG (A21020; 1:5,000; Beijing Zhongshan Jinqiao Biotechnology Co., Ltd.). Western blotting was quantified using the greyscale scanning method (Image J v1.48; National Institutes of Health, Bethesda, MD, USA).

Luciferase assay. Predictions for the potential binding sites of miR-590 in the 3'-UTR of Smad7 mRNA was performed using 3 different online programs: TargetScan (http://www. targetscan.org), microRNA.org (http://www.microrna.org) and the miRBase (http://www.mirbase.org). Fragments of the 3'-UTR of Smad7 mRNA, including the wild-type (wt) or the mutated (mt) site, was amplified and cloned downstream of the firefly luciferase in the pCDNA3.1-luciferase vector (Invitrogen; Thermo Fisher Scientific, Inc.). The mutation of the predicted binding site was produced using PCR-mediated site-specific mutation. For construct the vectors, the following primer sequences were used: wt 3'-UTR of Smad7 forward 5-CGCGGATCCCCGCGTGCGGAGGGGACAGA-3 and reverse 5-CCGCTCGAGGGAGTCCTTTCTCTCTCA AAGC-3. In the 3'UTR mutants, Smad7 3'UTR mutants from the 122-1,129 nucleotide sequence complementary to nucleotides 2-5 of miR-590 was mutated to the same sequence as that in miR-590 (from ATAAGCTA to TTATGCAA). For transfection, HUMSCs $\left(5 \times 10^{6}\right.$ cells/well) were seeded on 24 -well plates were transfected with $250 \mathrm{ng}$ pCDNA3.1-luciferase-Smad7 (wt and $\mathrm{mt}$ ), pRL-SV40-Renilla and pCDNA3.1-luciferase, and $50 \mathrm{nM}$ miR-590 mimic or negative control. After $24 \mathrm{~h}$, cells were lysed using $200 \mu \mathrm{l}$ radioimmunoprecipitation assay lysis buffer (Beyotime Institute of Biotechnology, Shanghai, China) at room temperature for $15 \mathrm{~min}$, centrifuged at $8,500 \mathrm{x} g$ at room temperature for $5 \mathrm{~min}$ to collect the supernatant and assayed by Dual-Luciferase Reporter assay kit (Promega Corporation, Madison, WI, USA), according to the manufacturer's protocol. Three independent experiments were performed.

5-Ethynyl-20-deoxyuridine (EdU) assay. For determination of cell proliferation, HUMSCs were suspended in MSC growth 


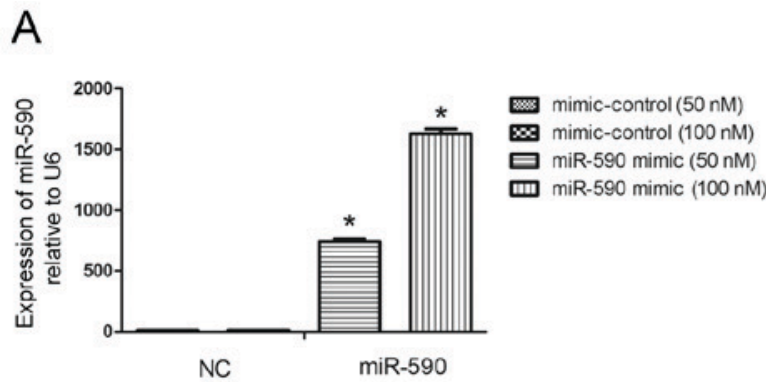

C

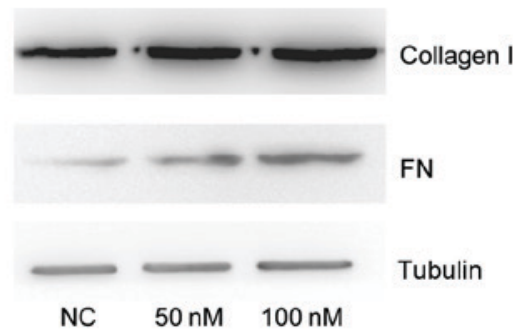

E

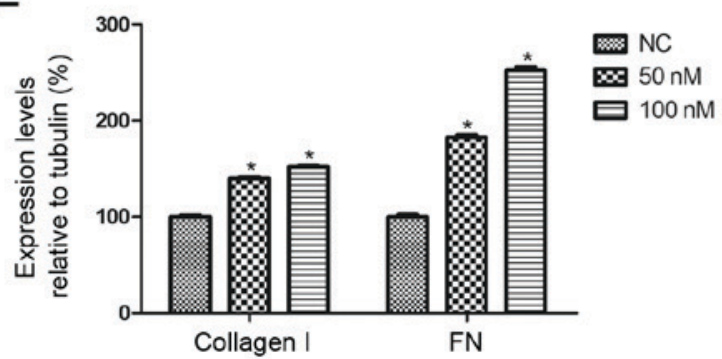

B

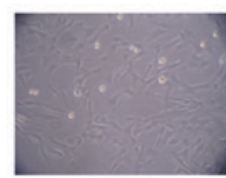

$\times 200$

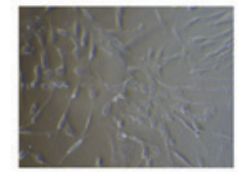

NC

D

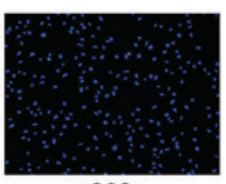

$\times 200$

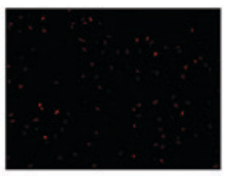

NC

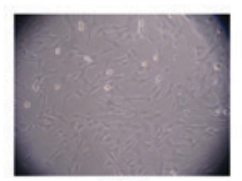

$\times 200$

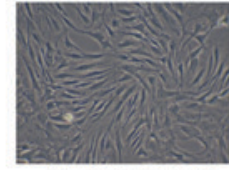

miR-590 (50 nM)

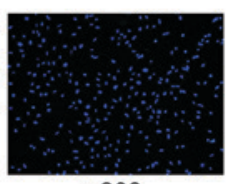

$\times 200$

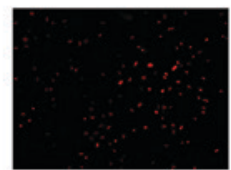

miR-590 (50 nM)

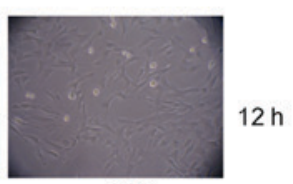

$\times 200$

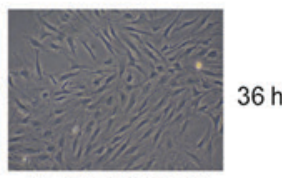

$\operatorname{miR}-590(100 \mathrm{nM})$

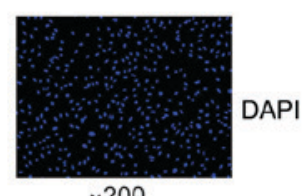

$\times 200$

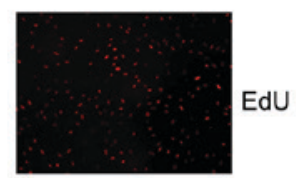

miR-590 (100 nM)

F

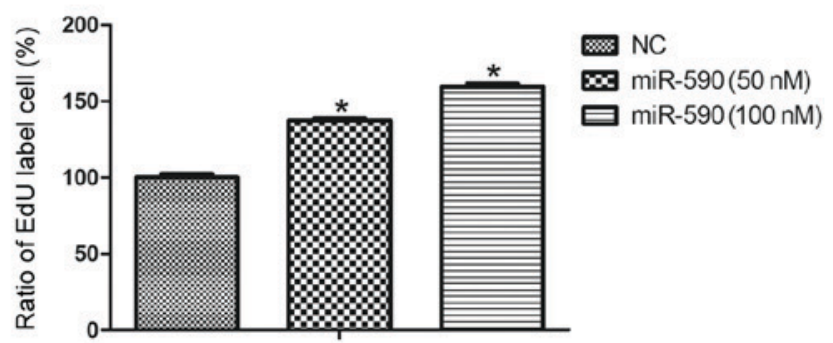

Figure 1. miR-590 transfection facilitates HUMSC proliferation and ECM deposit. (A) miR-590 mimic transfection resulted in a significant upregulation of

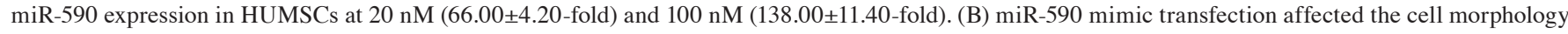
at $36 \mathrm{~h}$. (C) Western blot analysis of miR-590 mimic transfection effect on ECM protein expression levels after $48 \mathrm{~h}$. (D) Cell proliferation analysis of miR-590 mimic transfection effect on HUMSC proliferation at $36 \mathrm{~h}$. (E) Quantification of the effect of miR-590 mimic transfection on ECM protein expression. (F) Quantification of miR-590 mimic transfection effect on HUMSC proliferation. * $\mathrm{P}<0.05$ vs. NC. NC, negative control; HUMSC, human umbilical cord mesenchymal stem cell; ECM, extracellular matrix; FN, fibronectin; EdU, 5-ethynyl-20-deoxyuridine; miR, microRNA.

medium consisting of low-glucose Dulbecco's modified Eagle's medium containing $10 \%$ fetal bovine serum and cultured in 24 -well $\left(5 \times 10^{4}\right.$ cells $\left./ 100 \mu \mathrm{l}\right)$ plates 1 day before transfection. After 12 h, HUMSCs were transfected with miR-590 mimic (10 $\mathrm{nM})$, miRNA control $(10 \mathrm{nM})$, siRNA-Smad7 $(10 \mathrm{nM})$ or siRNA-control $(10 \mathrm{nM})$. Following transfection, cell proliferative ability was evaluated at $36 \mathrm{~h}$ using a EdU DNA Proliferation and Detection kit to label cellular DNA (Cell-Light 5-ethynyl-20-deoxyuridine Apollo 567 kit; Guangzhou RiboBio Co., Ltd.), according to the manufacturer's protocol. The percentage of HUMSCs proliferation was determined by counting the EdU proliferation cells in 1,000 cells. Subsequently, the fluorescence was used to detect DNA synthesis for cell proliferation at an ultraviolet light wavelength of $480 \mathrm{~nm}$. All experiments were performed in triplicate and independently.

Immunofluorescence microscopy. Following transfection with si-Smad7 or si-control $\left(5 \times 10^{6}\right.$ cells per well), cells cultured on glass coverslips were fixed with pre-chilled acetone for
$10 \mathrm{~min}$ at $-20^{\circ} \mathrm{C}$. Cells were permeabilized and blocked with $1 \%$ Triton X-100 (in PBS) and 5\% bovine serum albumin in PBS, respectively, each for $1 \mathrm{~h}$ at room temperature. The rabbit polyclonal antibody against collagen I (dilution, 1:500; ab209539; Abcam) was used to probe collagen I overnight at $4^{\circ} \mathrm{C}$ and goat anti-rabbit IgG-CruzFluor ${ }^{\mathrm{TM}} 594$ (dilution, 1:1,000; SC362282; Santa Cruz Biotechnology, Inc., Dallas, TX, USA) was used to determine rabbit $\mathrm{IgG}$ for $1 \mathrm{~h}$ at room temperature. Subsequently, the glass covers were washed with $1 \mathrm{X}$ PBS at room temperature for $3 \times 5 \mathrm{~min}$.

Finally, nuclei were stained with DAPI (dilution, 1:4,000; Santa Cruz Biotechnology, Inc.) at room temperature for $10 \mathrm{~min}$. The coverslips were immobilized on the glass slides using 50\% glycerol in PBS and viewed by fluorescence microscopy (ECLIPSE Ti-s; Nikon Corporation, Tokyo, Japan; 8-10 fields of view; magnification, x200) and images were captured with a SPOT Diagnostic charge-coupled device camera (Spot RT Color Diagnostic Instruments, Sterling Heights, MI, USA; $\sim 500$ cells per glass coverslip were counted under fluorescence microscopy). The intensity of fluorescence was evaluated using 
Image J v1.48 software following image capture (National Institutes of Health).

Statistical analysis. All experimental data were from triplicate experiments independently and data were expressed as a mean \pm standard deviation. GraphPad Prism (version 5; GraphPad Software, Inc., La Jolla, CA, USA) was used for data analysis. $\mathrm{P}<0.05$ was considered to indicate a statistically significant difference.

\section{Results}

miR-590 induces the proliferation of HUMSCs and ECM synthesis. The effects of miR-590 overexpression on HUMSCs, including cell morphological changes, proliferation and ECM synthesis, were evaluated. Following transfection with miR-590 mimic, the expression level of miR-590 in HUMSCs significantly increased at $50 \mathrm{nM}(66.00 \pm 4.20$-fold; $\mathrm{P}<0.0003)$ and $100 \mathrm{nM}(138.00 \pm 11.40$-fold; $\mathrm{P}<0.0001)$ compared with the negative control at $36 \mathrm{~h}$ (Fig. 1A), whereas cell morphology altered from the typical mesenchymal cell to fibroblast-like cell morphology (Fig. 1B). Western blot analysis indicated that there was increased expression levels of miR-590 leading to increases in the levels of collagen I and FN of $39.86 \pm 2.18$ and $82.99 \pm 3.16 \%$ for $50 \mathrm{nM}$, and $52.21 \pm 2.46$ and $152.03 \pm 4.58 \%$ for $100 \mathrm{nM}$, respectively, compared with the control group (Fig. 1C and E). Cell EdU analysis demonstrated that the proliferation of HUMSCs were increased to $38.15 \pm 2.14$ and $60.14 \pm 4.52 \%$ at $50 \mathrm{nM}$ and $100 \mathrm{nM}$, respectively, compared with the controls (Fig. 1D and F).

Predicting miR-590-binding sites in the 3'-UTR of Smad7 $m R N A$. To identify the downstream targets of miR-590, 3 independent online databases (TargetScan, microRNA.org and the miRBase) were selected to predict potential targets. In total, 110 putative mRNAs were predicted by the 3 databases (Fig. 2A) and among these Smad7 was of particular interest as previous studies have demonstrated its role in the cell proliferation and its effect on ECM (25). Following analysis of the sequence of Smad7 3'-UTR [1,520 base pairs (bp)], the only predicted binding site of miR-590 was identified at 1,122-1,129 bp (Fig. 2B).

Luciferase assay of miR-590 and Smad7 3'-UTR in HUMSCs. To determine whether Smad7 is a target of miR-590, a luciferase reporter vector was inserted with wt or $\mathrm{mt}$ Smad7 mRNA 3'-UTR, and this was cotransfected with miR-590 mimic. Overexpression of miR-590 in HUMSCs for $36 \mathrm{~h}$ significantly decreased $(\mathrm{P}<0.05)$ the luciferase activity of the luciferase reporter plasmid fused to wt Smad7 mRNA 3'-UTR (Fig. 2C), whereas the luciferase activity with mt Smad7 mRNA 3'-UTR was not affected (Fig. 2C).

Overexpression of miR-590 reduces Smad7 expression. To verify whether Smad7 is a functional upstream gene of miR-590, the mRNA and protein expression levels of Smad7 were analyzed. The miR-590 mimic and the negative control were transfected into HUMSCs. The expression of Smad7 at 36 and $48 \mathrm{~h}$ after transfection was detected using qPCR and western blot analysis for mRNA and protein, respectively.

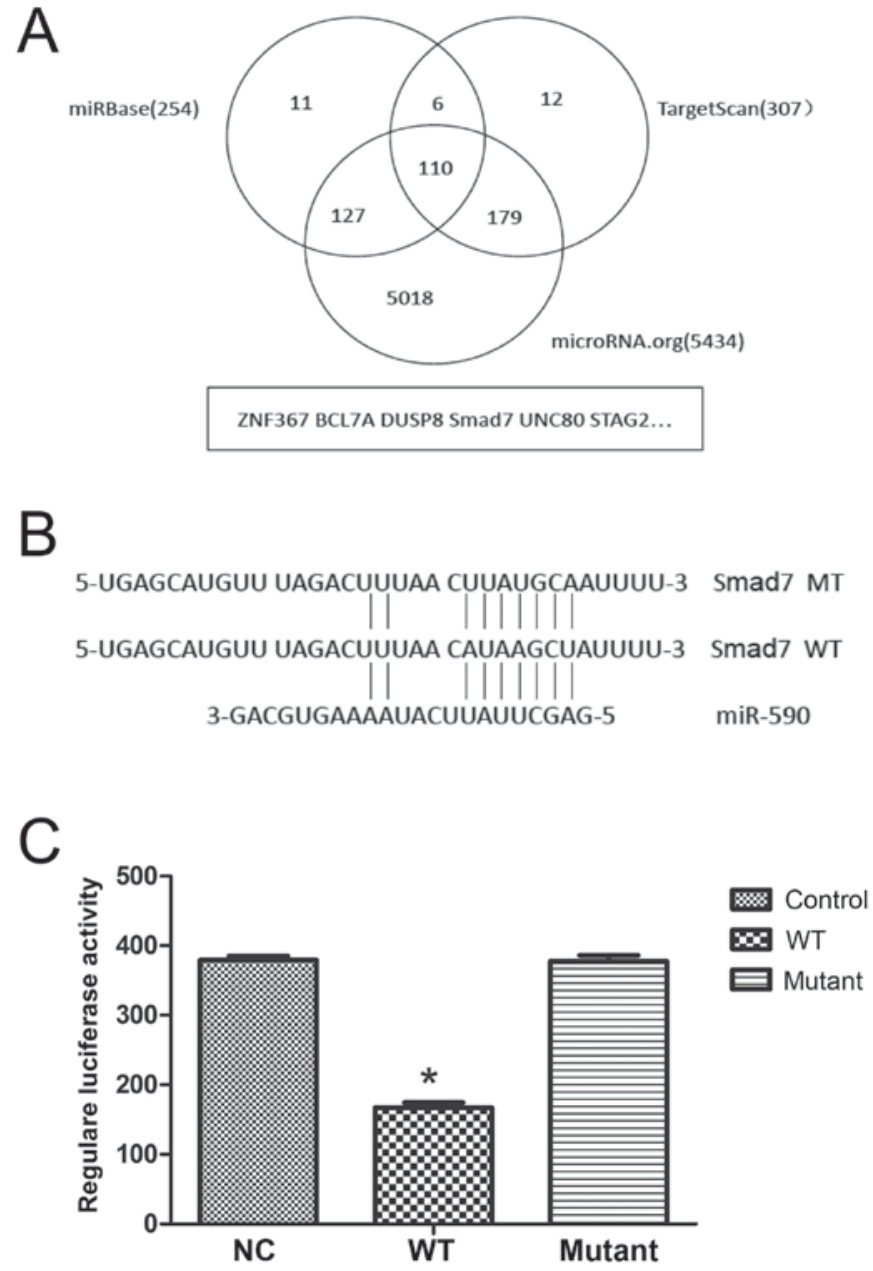

Figure 2. miR-590 directly binds to the 3'-UTR of Smad7 mRNA (A) Bioinformatic analyses predicted 110 potential target genes for miR-590 using 3 independent databases. (B) The predicted binding site of miR-590 in the 3'-UTR of Smad7 mRNA according to the miR-590 sequence and the fragment containing the mutated binding site was amplified. (C) In the pCDNA3.1-luciferase vector, the Smad7 mRNA 3'-UTR fragment containing the wild-type or mutated site was inserted downstream into the reporter gene. The plasmids were cotransfected with miR-590 mimic or mimic control and the relative luciferase activity, normalized by Renilla was significantly decreased using the wild-type sequence compared with that of the mutated site $(46.70 \pm 5.81 \%)$. ${ }^{*} \mathrm{P}<0.05$ vs. NC. NC, negative control; WT, wild-type; MT, mutated site; Smad7, Mothers Against Decapentaplegic Homolog 7; UTR, untranslated region; miR, microRNA; ZNF367, zinc finger 367; BCL7A, B-cell lymphoma 7A; DUSP8, dual-specificity phosphatase 8; UNC80, uncoordinated 80; STAG2, stromal antigen 2.

The overexpression of miR-590 downregulated the expression levels of Smad7 mRNA at $36 \mathrm{~h}(51.55 \pm 3.83 \%$; Fig. $3 \mathrm{~A})$. A similar effect was also identified on the protein level of Smad7 $(65.73 \pm 8.09 \%$ at $50 \mathrm{nM}$ and $72.36 \pm 9.38 \%$ at $100 \mathrm{nM}$; Fig. 3B and C).

Smad7 siRNA facilitates the proliferation of HUMSCs and ECM enhancement. The importance of Smad7 as a functional target for miR-590 was evaluated. HUMSCs were transfected with Smad7 siRNA or scramble control for 36 or $48 \mathrm{~h}$, and the mRNA $(43.72 \pm 6.48 \%$; Fig. $3 \mathrm{~A})$ and protein $(67.49 \pm 6.01 \%$; Fig. 3C) levels of Smad7 were significantly decreased using Smad7 siRNA. EdU assay and immunofluorescence microscopy were used to evaluate the proliferative ability and ECM 
A

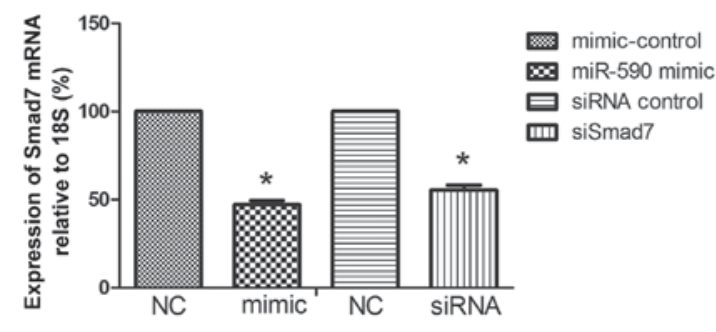

B

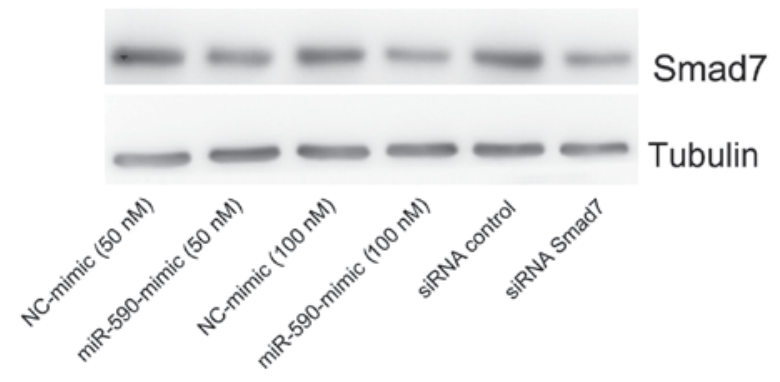

C

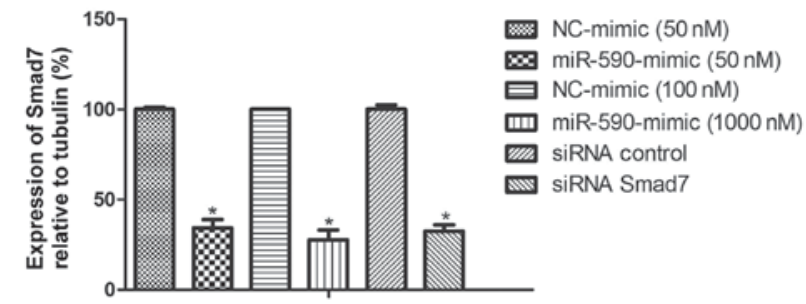

Figure 3. miR-590 transfection inhibits the mRNA and protein expression levels of Smad7. (A) miR-590 mimic and Smad7 siRNA transfection downregulated the expression of Smad7 mRNA to $51.55 \pm 3.83$ and $43.72 \pm 6.48 \%$, respectively. (B) Western blot analysis of miR-590 mimic or Smad7 siRNA transfection effect on Smad7. (C) Quantification of the effect of miR-590 mimic or Smad7 siRNA transfection on Smad7 protein expression. " $\mathrm{P}<0.05$ vs. NC. NC, negative control; miR, microRNA; Smad7, Mothers Against Decapentaplegic Homolog 7; si/siRNA, small interfering RNA.

expression in the HUMSCs, respectively. The proliferative ability of HUMSCs was increased to $30.17 \pm 2.31 \%$ compared with the control at $36 \mathrm{~h}$ (Fig. 4A). Immunofluorescence analysis identified decreased expression of Smad7 promoted collagen I upregulation and the proportion of collagen I increased to $67.86 \pm 8.18 \%$ compared with the control (Fig. 4B).

\section{Discussion}

Trauma is a predominant surgical outcome and therefore the problem of effective wound healing is a focus of investigation for researchers and clinicians. A series of pathological alterations, which are overlapping and interrelated, are involved in wound healing. For example, a number of cells and ECM components participate in the reconstruction (26). In tissue engineering, stem cell therapy is typically investigated. The theory of stem cell therapy, including cell-replacement (27) and ECM enhancement (28), may explain the underlying mechanisms involved in effective wound healing. miRNAs are a type of small non-coding RNA that regulate gene expression by directly binding to the $3^{\prime}$-UTR of mRNA (29). An increasing

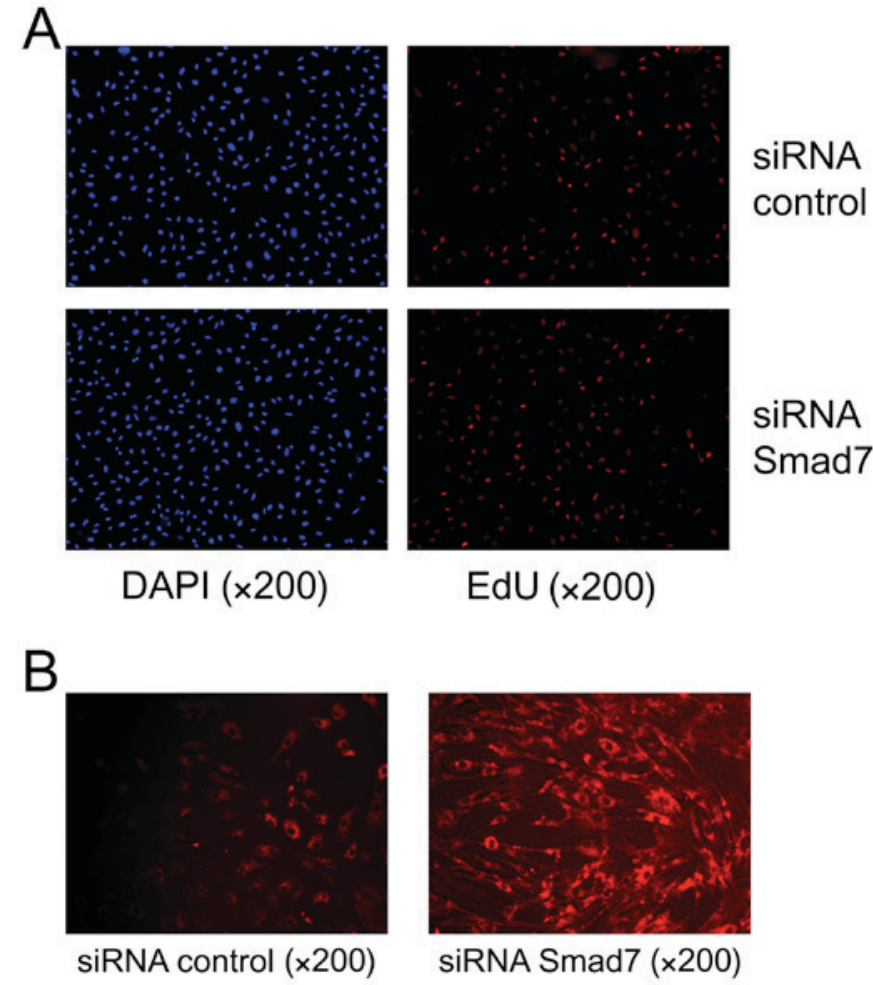

Figure 4. Smad7 siRNA induces HUMSC proliferation and ECM synthesis. (A) Cell proliferation analysis demonstrated that Smad7 siRNA transfection promoted HUMSC proliferation at $36 \mathrm{~h}(30.17 \pm 2.31 \%)$. (B) Immunofluorescence microscopy analysis identified that Smad7 siRNA transfection increases collagen I deposit at $48 \mathrm{~h}(67.86 \pm 8.18 \%)$ compared with the negative control. ${ }^{*} \mathrm{P}<0.05$ compared with $\mathrm{NC}$. NC, negative control; Smad7, Mothers Against Decapentaplegic Homolog 7; si/siRNA, small interfering RNA; HUMSC, human umbilical cord mesenchymal stem cell; ECM, extracellular matrix; EdU, 5-ethynyl-20-deoxyuridine.

number of studies have indicated that miRNAs have vital roles in numerous biological processes and in pathological disease, and previous studies have suggested that miRNAs participate in cell proliferation, apoptosis, differentiation and fibrosis $(29,30)$. However, the distinct role and underlying molecular mechanism of the involvement of miRNAs in stem cell therapy for the repair of trauma are yet to be elucidated.

In the past decades (2005 to 2015), miR-590 has been identified in numerous types of disease, including atherosclerosis (31), myocarditis (32), hepatocellular carcinoma (8) and renal cancer (7). In the present study, the effect of miR-590 on HUMSCs, including proliferation and ECM accumulation, were evaluated. Wound healing is a complex process involving the proliferation, migration, differentiation and ECM accumulation of local unwounded cells. In diabetic ulcers, a classic model of wound healing, miR-198 was significantly increased and inhibited migratory ability by targeting plasminogen activator urokinase, diaphanous related formin 1 and laminin subunit $\gamma 2$ (33). Considering rapid wound healing is critical and stem cell therapy is considered promising, the investigation of miR-590 effect on wound healing was required. In the present study, miR-590 overexpression promoted cell proliferation in HUMSCs and ECM enhancement was demonstrated using western blotting and immunofluorescence microscopy. To explain the effects of miR-590, the potential targets of miR-590 were identified using bioinformatic analyses. 
Notably, Smad7 was identified as one of the potential targets and selected for further investigation. Further experiments identified that the expression level of $\mathrm{Smad} 7$ is repressed at the mRNA and protein level by miR-590 overexpression. According to the luciferase assay, one predicted binding site of miR-590 on 3'-UTR of Smad7 mRNA was considered to be a functional site. On the basis of the identification of Smad7 as a novel target of miR-590, the function of Smad7 mRNA in cell proliferation and ECM synthesis were assessed. The transfection of Smad7 siRNA resulted in a similar stimulation of cell proliferation and ECM promotion in HUMSCs compared with miR-590 overexpression.

In conclusion, the results of the present study suggested that miR-590 induces stem cell proliferation by targeting Smad7. Therefore, future studies may be able to determine whether miR-590 regulates other genes for the promotion of stem cell differentiation or migration.

\section{Acknowledgements}

The present study was supported by the National Natural Science Foundation of China (grant no. 81302540).

\section{References}

1. Lazarus GS, Cooper DM, Knighton DR, Percoraro RE, Rodeheaver G and Robson MC: Definitions and guidelines for assessment of wounds and evaluation of healing. Wound Repair Regen 2: 165-170, 1994.

2. Boateng JS, Matthews KH, Stevens HN and Eccleston GM: Wound healing dressings and drug delivery systems: A review. J Pharm Sci 97: 2892-2923, 2008.

3. Seshareddy K, Troyer D and Weiss ML: Method to isolate mesenchymal-like cells from Wharton's Jelly of umbilical cord. Methods Cell Biol 86: 101-119, 2008.

4. Banerjee J, Chan YC and Sen CK: MicroRNAs in skin and wound healing. Physiol Genomics 43: 543-556, 2011.

5. Sand M, Gambichler T, Sand D, Skrygan M, Altmeyer P and Bechara FG: MicroRNAs and the skin: Tiny players in the body's largest organ. J Dermatol Sci 53: 169-175, 2009.

6. Stefani G and Slack FJ: Small non-coding RNAs in animal development. Nat Rev Mol Cell Biol 9: 219-230, 2008.

7. Xiao X, Tang C, Xiao S, Fu C and Yu P: Enhancement of proliferation and invasion by microRNA-590-5p via targeting PBRM1 in clear cell renal carcinoma cells. Oncol Res 20: 537-544, 2013.

8. Jiang X, Xiang G, Wang Y, Zhang L, Yang X, Cao L, Peng H, Xue $\mathrm{P}$ and Chen D: MicroRNA-590-5p regulates proliferation and invasion in human hepatocellular carcinoma cells by targeting TGF-b RII. Mol Cells 33: 545-551, 2012.

9. Shan X, Miao Y, Fan R, Qian H, Chen P, Liu H, Yan X, Li J and Zhou F: MiR-590-5P inhibits growth of HepG2 Cells via decrease of S100A10 expression and inhibition of the Wnt pathway. Int J Mol Sci 14: 8556-8569, 2013.

10. Chu Y, Ouyang Y, Wang F, Zheng A, Bai L, Han L, Chen Y and Wang H: MicroRNA-590 promotes cervical cancer cell growth and invasion by targeting CHL1. J Cell Biochem 115: 847-853, 2014.

11. Li K, Li Z, Zhao N, Xu Y, Liu Y, Zhou Y, Shang D, Qiu F, Zhang R, Chang $\mathrm{Z}$ and $\mathrm{Xu}$ Y: Functional analysis of microRNA and transcription factor synergistic regulatory network based on identifying regulatory motifs in non-small cell lung cancer. BMC Syst Biol 7: 122, 2013

12. Lee S, Yu KR, Ryu YS, Oh YS, Hong IS, Kim HS, Lee JY, Kim S, Seo KW and Kang KS: miR-543 and miR-590-3p regulate human mesenchymal stem cell aging via direct targeting of AIMP3/p18. Age (Dordr) 36: 9724, 2014.

13. Shan H, Zhang Y, Lu Y, Zhang Y, Pan Z, Cai B, Wang N, Li X, Feng T, Hong Y and Yang B: Downregulation of miR-133 and miR-590 contributes to nicotine-induced atrial remodelling in canines. Cardiovasc Res 83: 465-472, 2009.
14. Kavsak P, Rasmussen RK, Causing CG, Bonni S, Zhu H, Thomsen GH and Wrana JL: Smad7 binds to Smurf2 to form an E3 ubiquitin ligase that targets the TGF beta receptor for degradation. Mol Cell 6: 1365-1375, 2000.

15. Fukasawa H, Yamamoto T, Togawa A, Ohashi N, Fujigaki Y, Oda T, Uchida C, Kitagawa K, Hattori T, Suzuki S, et al: Down-regulation of Smad7 expression by ubiquitin-dependent degradation contributes to renal fibrosis in obstructive nephropathy in mice. Proc Natl Acad Sci USA 101: 8687-8692, 2004.

16. Afrakhte M, Morén A, Jossan S, Itoh S, Sampath K, Westermark B, Heldin CH, Heldin NE and ten Dijke P: Induction of inhibitory Smad6 and Smad7 mRNA by TGF-beta family members. Biochem Biophys Res Commun 249: 505-511, 1998.

17. Jia J, Feng X, Xu W, Yang S, Zhang Q, Liu X, Feng Y and Dai Z: MiR-17-5p modulates osteoblastic differentiation and cell proliferation by targeting SMAD7 in non-traumatic osteonecrosis. Exp Mol Med 46: e107, 2014.

18. Liu G, Friggeri A, Yang Y, Milosevic J, Ding Q, Thannickal VJ, Kaminski N and Abraham E: miR-21 mediates fibrogenic activation of pulmonary fibroblasts and lung fibrosis. J Exp Med 207: $1589-1597,2010$

19. Chen G, Cao S, Liu F and Liu Y: miR-195 plays a role in steroid resistance of ulcerative colitis by targeting Smad7. Biochem J 471: 357-367, 2015

20. Zhu B, Wei XX, Wang TB, Zhou YC, Liu AM and Zhang GW: Increased miR-16 expression induced by hepatitis $C$ virus infection promotes liver fibrosis through downregulation of hepatocyte growth factor and Smad7. Arch Virol 160: 2043-2050, 2015.

21. Wang ZH, Zhang QS, Duan YL, Zhang JL, Li GF and Zheng DL: TGF- $\beta$ induced miR-132 enhances the activation of TGF- $\beta$ signaling through inhibiting SMAD7 expression in glioma cells. Biochem Biophys Res Commu 463: 187-192, 2015.

22. Liu N, Jiao T, Huang Y, Liu W, Li Z and Ye X: Hepatitis B virus regulates apoptosis and tumorigenesis through the microRNA-15a-Smad7-transforming growth factor $\beta$ pathway. J Virol 89: 2739-2749, 2015

23. Liu J, Yao W, Yao Y, Du X, Zhou J, Ma B, Liu H, Li Q and Pan Z: miR-92a inhibits porcine ovarian granulosa cell apoptosis by targeting Smad7 gene. FEBS Lett 588: 4497-4503, 2014.

24. Trang NV, Choisy M, Nakagomi T, Chinh NT, Doan YH, Yamashiro T, Bryant JE, Nakagomi O and Anh DD: Determination of cut-off cycle threshold values in routine RT-PCR assays to assist differential diagnosis of norovirusin children hospitalized for acute gastroenteritis. Epidemiol Infect 143: 3292-3299, 2015.

25. Su Y, Yang CY, Li Z, Xu F, Zhang L, Wang F and Zhao S: Smad7 siRNA inhibit expression of extracellular matrix intrabecular meshwork cells treated with TGF- $\beta 2$. Mol Vis 18: 1881-1884, 2012.

26. Shakespeare P: Burn wound healing and skin substitutes. Burns 27: 517-522, 2001.

27. Newman RE, Yoo D, LeRoux MA and Danilkovitch-Miagkova A: Treatment of inflammatory diseases with mesenchymal stem cells. Inflamm Allergy Drug Targets 8: 110-123, 2009.

28. Arno AI, Amini-Nik S, Blit PH, Al-Shehab M, Belo C, Herer E, Tien $\mathrm{CH}$ and Jeschke MG: Human Wharton's jelly-mesenchymal stem cells promote skin wound healing through paracrine signaling. Stem Cell Res Ther 5: 28, 2014.

29. Bushati N and Cohen SM: microRNA functions. Annu Rev Cell Dev Biol 23: 175-205, 2007

30. Garzon R, Calin GA and Croce CM: MicroRNAs in cancer. Annu Rev Med 60: 167-179, 2009.

31. He PP, OuYang XP, Li Y, Lv YC, Wang ZB, Yao F, Xie W, Tan YL, Li L, Zhang M, et al: MicroRNA-590 inhibits lipoprotein lipase expression and prevents atherosclerosis in apoE knockout mice. PLoS One 10: e0138788, 2015.

32. Zhao S, Yang G, Liu PN, Deng YY, Zhao Z, Sun T, Zhuo XZ, Liu JH, Tian Y, Zhou J, et al: miR-590-3p is a novel MicroRNA in myocarditis by targeting nuclear factor Kappa-B in vivo. Cardiology 132: 182-188, 2015.

33. Sundaram GM, Common JE, Gopal FE, Srikanta S, Lakshman K, Lunny DP, Lim TC, Tanavde V, Lane EB and Sampath P: 'See-saw' expression of microRNA-198 and FSTL1 from a single transcript in wound healing. Nature 495: 103-106, 2013. 